


\section{Critique sémiotique du populisme}

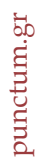

BY: Eric Landowski

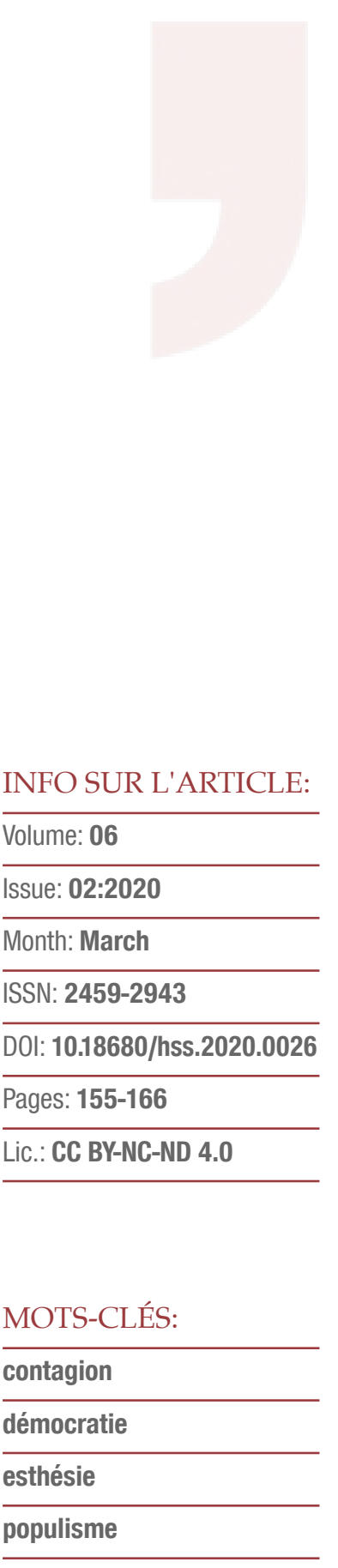

RÉSUMÉ

omment rendre compte de la faveur dont jouissent aujourd'hui tant de leaders populistes dans le monde? Les explications des politologues combinent généralement trois facteurs contextuels: le pouvoir de séduction des idéologies 'anti-système' (facteur de base) est renforcé par l'impact de la crise socio-économique (deuxième facteur) et par diverses données conjoncturelles - scandales, attentats, catastrophes naturelles, etc. (troisième facteur). Reste un facteur moins exploré, à savoir la force des liens d'ordre sensible (ou 'esthésique') que ce type de leaders établissent avec leurs partisans, presque indépendamment de ce qu'ils ont de précis à proposer en termes de politiques publiques. Cerner les conditions et les effets de telles relations revient à proposer (conformément à un des principes qui fondent l'approche sémiotique) une explication du phénomène dans son immanence, c'est-à-dire focalisée sur l'analyse des rapports de contagion thymique qui se nouent entre l'hexis du tribun populiste et chacun de ses partisans. D'où résulte un simulacre de démocratie 'directe' (par opposition à 'représentative') qui, par interdéfinition, trouve sa place dans une typologie sémiotique des régimes politiques.

\section{Expliquer le populisme?}

De la Grande-Bretagne à l'Italie, de la Hongrie à la France ou aux Pays-Bas, du Brésil aux Etats-Unis, pratiquement tous les pays occidentaux ont été affectés ces dernières années à des de-

\footnotetext{
${ }^{1}$ Une version préliminaire du présent article est parue en lithuanien: "Semiotinè populizmo kritika" (trad. Nijolé Kersyté), Semiotika 15, 2020.
} 
grés divers par la vague 'populiste.' Sans discuter de la justesse ou de l'impropriété de ce terme au regard de la théorie politique, nous le prendrons ici tel qu'on le trouve aujourd'hui en circulation: comme une étiquette appliquée à divers courants à forte teneur nationaliste qui ont en commun de se présenter comme les défenseurs des intérêts du 'peuple' (sous-entendu, exclusivement celui de chez soi) et de préconiser ou de prendre à cet effet des mesures qui non seulement vont à l'encontre des principes démocratiques mais qui de surcroît menacent l'équilibre des relations internationales. A ce titre, le phénomène mérite l'analyse et appelle la critique, notamment du point de vue sémiotique. Il est vrai qu'il existe tant d'études en la matière que tout paraît avoir déjà été dit. Un regard sémiotique peut-il apporter quelque chose de plus?

Les interprétations des politologues combinent généralement trois facteurs explicatifs: l'actuel 'raz-de-marée' tiendrait à ce que le pouvoir de séduction de l'idéologie (facteur de base) que diffusent les mouvements en question est renforcé de nos jours par l'impact négatif de l'évolution socio-économique (deuxième facteur) sur la clientèle politique visée, et par diverses données conjoncturelles - scandales, 'affaires,' attentats ou catastrophes naturelles (troisième facteur) - coïncidant avec les moments de leurs plus grands succès électoraux. Ces interprétations passent en revanche sous silence un quatrième facteur, dont la sémiotique a particulièrement vocation à éclairer les mécanismes. Cet élément susceptible d'expliquer directement la ferveur que suscitent les Salvini, Johnson, Bolsonaro, Le Pen, Orban, Trump et autres, c'est la forme de leur mise en scène, la manière, en grande partie commune à eux tous, dont ils se présentent publiquement, et le type de rapports qui en résultent entre eux et leurs partisans - cela, certes, non pas indépendamment de ce qu'ils ont à proposer mais pour ainsi dire en deçà.

Cerner la spécificité, les conditions et les effets des liens quasi interpersonnels, immédiatement et intimement éprouvés, qui se nouent entre les leaders populistes et leurs auditoires, ce serait (conformément à l'un des principes hjelmsleviens qui fondent l'approche sémiotique) rendre compte du phénomène par une explication en immanence, c'est-à-dire fondée sur des données constitutives de l'objet même, sur la relation même entre la source et la cible des pratiques de mise en scène 'populiste.' Par là, ce serait aussi apporter des éléments de compréhension complémentaires par rapport à ceux que fournissent les approches les plus courantes puisque, de leur côté, elles ont pour trait commun de faire au contraire appel à des données contextuelles, qu'il s'agisse de la sociologie de l'électorat, de facteurs structurels comme le chômage ou l'immigration, ou circonstantiels comme les 'affaires,' les attentats ou d'autres accidents. 


\section{Crise de confiance}

Prenons néanmoins comme point de départ la thèse principale qui se dégage des études sociologiques ou politologiques disponibles: les supporters des mouvements populistes seraient tous des 'perdants de la mondialisation' et des 'exclus de la révolution numérique.' Leur vote serait, autrement dit, ici et là l'expression d'un même genre de frustrations liées au changement technologique et économique global, partout aggravées par un même ressentiment à l'égard d"élites' jugées incapables ou même supposées peu soucieuses de résoudre les problèmes engendrés par ces évolutions.

En termes de sémiotique narrative, on a là affaire à une crise 'fiduciaire': c'est la perte de confiance dans le personnel politique et dans les institutions qui a éloigné des formes de participation politique traditionnelles une masse de citoyens victimes de la conjoncture mondiale et a conduit une partie d'entre eux vers l'abstention tout en poussant les plus indignés, les plus révoltés ou les plus résolus du côté de personnalités rejetées par la 'classe politique.' Pour des électeurs qui rêvent d'écarter du pouvoir les représentants d'un 'establishment' tenu pour responsable de leur injuste sort, quoi de plus logique en effet que de se rallier à des 'outsiders' qui s'en proclament les ennemis, au nom du 'peuple' justement?

Cette vision en noir et blanc et la logique d'exclusion qu'elle suppose ne paraissent pourtant pas suffisantes. Mis à part certains cas de ralliement quasi mécanique à un candidat par simple rejet de son adversaire, il ne suffit généralement pas de se défier d'un camp pour se rallier au camp opposé. Il y faut en plus un minimum de motifs positifs, un élan, une séduction, certaines affinités assez fortement ressenties pour attirer. Or, si on voit bien comment la confiance se perd (par exemple comment celle qui a été placée dans un dirigeant peut être rompue pour cause de non-respect de ses engagements), il est moins simple de discerner comment elle se gagne. Surtout quand il s'agit, comme cela arrive de plus en plus fréquemment, de l'accorder non pas à des professionnels de la politique connus de longue date mais à des postulants nouveaux, issus de la 'société civile' et de ce fait restés trop éloignés des fonctions de représentation et de gouvernement pour qu'on puisse imaginer ce dont ils seraient capables, en bien ou en mal, s'ils accédaient au pouvoir.

Pourtant, c'est précisément de cette sorte de virginité politique que se prévalent la plupart des candidats populistes. Leur art consiste à tourner en vertu leur relatif manque d'expérience, leur non-professionnalisme et leur distance par rapport aux mœurs, au langage, à l'esprit même des sphères politiques établies. Cette forme d'amateurisme qui serait rédhibitoire pour tout autre type de carrière n'empêche pas, de nos jours - au contraire - de devenir maire d'une grande métropole, premier ministre ou chef d'Etat. De fait, se présenter comme politiquement novice, comme quelqu'un qui n'a jamais été mêlé aux intrigues de la 'politique politicienne,' qui ne dépend d'aucun 
parti, qui n'a aucune part de responsabilité dans les fautes des gouvernements précédents, c'est se donner l'allure d'un brave citoyen 'comme nous,' étranger aux compromis et aux mensonges du 'système' mais en revanche victime ou, pour le moins, observateur outré par ses errements, ses injustices, ses tromperies.

Cette insistance sur l'appartenance au monde des humbles par opposition à celui des puissants, au 'peuple' par opposition aux privilégiés, et en même temps (car dans la bouche des populistes cela va de pair) sur l'enracinement dans la mère patrie, par opposition à des étrangers, immigrés ou réfugiés, vus comme des accapareurs d'emplois et des promoteurs d'insécurité, tout cela va dans le même sens. De tels gages de solidarité et de complicité avec les indignés sont apparemment, pour ce type paradoxal de politiciens anti-politiciens, les meilleurs moyens de construire l'image de candidats dignes de confiance. A les voir et à les entendre, tout donne à croire qu'une fois au pouvoir ils ne risqueront de trahir ni les victimes de la globalisation ni les 'laissés-pourcompte' de la fête digitale qui mettent en eux leurs espoirs de revanche.

\section{Vers l'esthésie}

Si la confiance qu'inspirent les leaders populistes repose sur le type d'éléments qu'on vient d'évoquer - la possession d'un 'casier politique vierge,' la position 'hors-système,' la qualité de 'vrai autochtone,' ${ }^{2}$ — alors il faut en bonne logique admettre qu'elle n'a pas pour condition nécessaire une compréhension approfondie de la politique qu'ils s'engagent à mener sur le plan économique, social, international, etc. Peut-être d'ailleurs une bonne partie de leurs électeurs sont-ils ou se croient-ils encore moins que d'autres à même de juger en ces matières souvent présentées comme très techniques. Peut-être même n'en ont-ils pas le souci. Le fait que cela ne les empêche pas de savoir qui applaudir renforce l'idée que le contenu précis des programmes politiques n'est pas le critère principal de leur choix: l'image de la personne l'emporte sur le détail de ses propositions.

Mais l'image' ne suffit pas. Cette notion implique une distance entre celui qui regarde et ce qu'il voit. Or, en politique, il ne s'agit pas toujours - pas forcément - de regarder et de juger pour, ensuite, décider. Comme la sympathie ou l'antipathie, l'adhésion politique peut aussi être de l'ordre de l'élan spontané, 'sans raison.' Ou plutôt, il peut aussi s'agir d'une impulsion qui a bien certaines 'raisons,' mais des raisons éprouvées plutôt que raisonnées, autrement dit qui reposent sur des sentiments d'affinité immédiate relevant d'un plan de pertinence distinct du plan cognitif. Ce ressenti, cet éprouvé, on les voit se développer face au tribun populiste au moins selon deux modes complémentaires.

\footnotetext{
${ }^{2}$ En universalisant le nom du parti populiste finlandais, les Vrais Finlandais.
} 
D'un côté, sur le mode d'humeurs partagées. Il s'agit là d'une composante essentielle de l'ethos populiste: c'est la xénophobie — ni un parti pris idéologique ni une option réfléchie mais une compulsion de rejet à base émotionnelle et même somatique, 'viscérale.' Pour un leader sans scrupule, donner à ses supporters l'occasion de communier dans la haine des autres, voilà un puissant moyen de rassemblement autour de sa personne. D'où l'intérêt des démagogues à exciter, outre l'exécration des 'élites,' les pulsions à connotation raciale, même si elles ne sont nullement l'apanage des milieux 'populaires.' Lorsqu'elle a pour ressort le partage du sentiment de différer ensemble face aux 'envahisseurs' au point de 'ne pas pouvoir les sentir' (ce qui veut dire les exécrer en tant que corps jusqu'au fond de son propre corps), la dévotion au chef ne tient à rien d'autre qu'à un pur sentir, le plus brut et le plus brutal. Sur ce point, le modèle historique insurpassable est le discours de meeting hitlérien. Là, ce qui compte est moins l'argumentation qui cherche à motiver la haine (en tant que passion fondée sur des raisons de haïr) que le ton, le geste, le rythme, la fantastique dynamique corporelle $\mathrm{d}^{\prime}$ un orateur capable de faire partager la haine, de la faire vivre en tant que motion somatique contagieusement induite.

Mais d'un autre côté, la confiance se fonde aussi, plus pacifiquement, sur l'exaltation des similitudes entre soi. Le plus élémentaire dans cet ordre d'idée, c'est la certitude de se trouver entre proches, du même monde et du même bord. Or l'un des principaux talents des leaders populistes est précisément de savoir se faire passer pour proches de leur clientèle. Pour beaucoup d'entre eux, une présence continuelle sur les réseaux sociaux est devenue, on le sait, le moyen le plus efficace d'entretenir avec leur clientèle un lien de familiarité pour ainsi dire intime. Parallèlement, en montrant qu'ils se comportent 'comme tout le monde' dans la vie de tous les jours, leur mise en scène sur les grands et petits écrans atteste à chaque instant qu'ils partagent 'nos soucis,' et que s'ils les partagent, c'est parce qu' eux aussi ils sont 'du peuple.' S'exprimer avec le même langage familier que 'vous et moi' en sera la preuve. Certains vont même plus loin, passant de la familiarité à une grossièreté calculée pour scandaliser le 'beau monde' (les gens ' $d$ 'en haut') tout en faisant jubiler leurs fidèles (les gens soi-disant 'd'en bas'): sur la scène politique, savoir-faire scandale par une parole dont l'insolence gage la 'vérité' fait aussi partie des compétences du bouffon. ${ }^{3}$

Fondée sur de tels éléments, la crédibilité d'un leader ne dépend ni de la faisabilité de ses projets ni de la cohérence ou de la véracité de ses propos. Puisque c'est une sympathie à fleur de peau pour la personne même qui emporte l'adhésion des supporters à ce qu'il énonce, ni la démagogie de ses promesses ni la fausseté éventuelle de ses assertions ne peuvent lui nuire. Pour ceux qu'il fascine, il ne peut y avoir de sa part ni

\footnotetext{
${ }^{3}$ Bouffon, clown, histrion? Falstaff, Ubu, Coluche... Trump, Johnson, Bolsonaro, Grillo, Le Pen... toute une lignée professionnelle, de la scène de théâtre à la scène politique, atteste que ces termes n'ont rien de déplacé. Sur le bon usage de la grossièreté en politique, cf. Landowski (1997, chap. 7, 'Régimes de présence et formes de popularité').
} 
discours mensonger ni parole délirante. Aucune réfutation, aucun fact-checking ne pourront donc être crus ni même entendus. Non pas qu'on les juge à leur tour faux mais tout simplement parce qu'aux yeux des fidèles ils ne sont pas pertinents en tant qu'actes de langage : par définition, contre la parole du dépositaire d'une confiance aveugle, aucune argutie n'est recevable.

On a là le point d'aboutissement d'un changement radical d'attitude épistémique. Une forme de discursivité faite de rigueur argumentative a perdu sa prégnance tandis qu'à côté d'elle mais aussi, en grande partie, à sa place, le sensible, 1"esthésique,' en est venu à jouer un rôle prépondérant. ${ }^{4}$ Cela ne veut certes pas dire que le pouvoir d'incantation des orateurs populistes soit à lui seul suffisant pour gagner les suffrages de n'importe quel auditoire. Leurs discours ne peuvent certes avoir prise que sur ceux dont la vision du monde les prédispose à croire aux solutions miraculeuses que promet le démagogue vu comme un sauveur. Sans un minimum de conditions de cet ordre, qui par nature relèvent du plan cognitif, le charme exercé sur le plan esthésique n'opèrerait pas. Mais pour peu qu'elles soient réunies, alors un timbre de voix, une expression physionomique, une gestualité singulière, un ton, un rythme, la vertu contagieuse d'une forte 'présence' peuvent servir de points de cristallisation pour la formation de liens éprouvés comme si c'était de personne à personne et les yeux dans les yeux. ${ }^{5}$

\section{Régimes sémiotiques et régimes politiques}

Pendant longtemps, dans les régions du monde actuellement en proie au populisme, a régné une parfaite concordance entre le régime juridico-politique de la représentation, ou 'démocratie représentative,' et le régime sémiotique dit de la manipulation, où des procédures de persuasion réciproque débouchent sur des accords entre sujets. L'électeur-citoyen, tout comme le héros du récit sémiotique canonique, était, au moins en théorie, un sujet autonome, guidé par un principe d'intentionnalité, doué de volonté et de raison. Conscient de lui-même et de ses intérêts, il décidait de tout en connaissance de cause, sachant peser le pour et le contre et estimer la juste valeur des 'valeurs.' Même ses emportements passionnels obéissaient à une forme de rationalité qui les rendait sensés et grosso modo prévisibles. L'homo politicus (et son frère l'homo æconomicus), à l'instar de l'homo semioticus, était essentiellement un actant-sujet cognitivement compétent - un esprit 'intelligent.'

Puis, dans la théorie, est apparu non pas son contraire (un agent sans discernement ni volonté ne serait plus en effet, à proprement parler, un 'sujet') mais son complémen-

\footnotetext{
${ }^{4}$ Sur la notion d'esthésie, cf. Greimas (1987).

${ }^{5}$ Sur l'intimité paradoxale du rapport médiatique, cf. Landowski (2004, chap. 10).
} 
taire : un sujet incarné, perméable, corps et âme, aux qualités esthésiques du monde et des présences humaines qui occupent son voisinage - en un mot, un sujet 'sensible.' L'intégration du principe de sensibilité ainsi reconnu à l'intérieur d'un modèle interactionnel élargi a conduit à la mise en lumière, à côté du régime de la manipulation fondé sur le principe d'intentionnalié, d'un régime de sens et d'interaction tout à fait différent et jusqu'alors sémiotiquenent inconnu, celui de l'ajustement. Mais à la manipulation et à l'ajustement, le modèle interactionnel que nous proposons articule deux autres régimes: celui de la programmation, fondé sur un principe de régularité, et celui de l'accident, caractérisé au contraire par l'absence de toute régularité, autrement dit régi par un principe d'aléa. ${ }^{6}$

Séparément ou combinés les uns aux autres, ces divers régimes permettent de rendre compte de la dimension signifiante de l'interaction dans toutes sortes de domaines. Dans le domaine qui nous intéresse ici, à chacun d'entre eux correspond un type de régime politique déterminé:

\begin{tabular}{|l|l|l|l|}
\hline $\begin{array}{l}\text { Principes de signifiance } \\
\text { et d'interaction }\end{array}$ & La régularité & L'imprévisibilité \\
\hline Régimes d'interaction & La programmation & & L'assentiment à l'aléa \\
\hline Régimes politiques & Totalitarisme & Absolutisme \\
\hline Principes de signifiance & La distinctivité & & La sensibilité \\
\hline Principes d'interaction & L'intentionnalité & & La disponibilité \\
\hline Régimes d'interaction & La manipulation & & L'ajustement \\
\hline Régimes politiques & $\begin{array}{l}\text { Démocratie } \\
\text { représentative }\end{array}$ & $\begin{array}{l}\text { Démocratie directe ou } \\
\text { démagogie populiste }\end{array}$ \\
\hline
\end{tabular}

Les totalitarismes sont des régimes politiques où la collectivité tout entière est soumise à des principes rigides, des régularités théoriquement immuables, en général appuyées sur une prétendue connaissance scientifique des 'lois' de la société et de l'économie. Les interactions entre agents sont en conséquence encadrées par des réglementations et orientées par des planifications qui ont pour effet de réduire les comportements individuels et collectifs à l'exécution d'algorithmes préétablis: en termes

${ }^{6}$ Présenté initialement dans Les interactions risquées, Limoges, PULIM, 2005, ce modèle a été actualisé in Landowski (2019) (https:/ / bit.ly/3b1U2t0). 
sémiotiques, c'est la syntaxe du régime interactionnel de la programmation qui est ainsi mise en œuvre. Du corporatisme de la France de Vichy, sous l'occupation allemande, à la mise en ordre sociétale nazie, fasciste ou stalinienne, les exemples historiques de ce type de régimes largement soutenus par les masses, et, en ce sens, 'populaires,' sont trop connus pour qu'il soit nécessaire d'entrer dans des détails. On doit en revanche se demander si les formes actuelles de normalisation à grande échelle rendues possibles par le développement des instruments de surveillance informatique ne vont pas tout doucement dans la même direction, celle d'un totalitarisme certes d'allure plus 'soft' mais d'autant plus efficace qu'il est mieux accepté sinon même désiré par la masse de la population ('populiste' comprise).

Non moins rigides et contraignants, bien que d'une tout autre manière, les absolutismes placent les sujets politiques sous la dépendance d'une autorité toute puissante dont les décisions ne dépendent que de son propre caprice: c'est la volonté souveraine et arbitraire du prince qui détermine le sort de tout un chacun sous la forme de diktats aussi difficiles à anticiper que les accidents de la vie ordinaire. L'autorité ne se fonde plus alors sur une 'science' de la société mais sur un dogme, tel celui de la transcendance du pouvoir 'de droit divin' investi dans l'autorité suprême du temps des anciennes monarchies. Qu'il s'agisse du monarque au sens propre ou, à l'époque moderne, de figures de pouvoir investies d'une sacralité laïque (depuis le Petit Père des Peuples ou le Grand Timonier d'antan jusqu'aux divers 'hommes providentiels' actuellement révérés par les populistes un peu partout dans le monde), les décisions que prennent de tels dirigeants relèvent d'un pouvoir quasi-discrétionnaire. Par suite, leurs choix ne sont guère plus prévisibles que ne le sont les 'décrets' du hasard. Le régime sémiotique qui en résulte pour les sujets est donc celui de l'accident inévitable - mais aussi, en même temps, bien souvent, celui de l'assentiment à l'arbitraire de l'autorité suprême: le plus généralement, face à l'absolu du pouvoir, que peut-on en effet, si ce n'est y consentir comme à une fatalité? Si le 'culte de la personnalité' a constitué naguère, dans divers pays, une des formes les plus notoires d'un tel assentiment, la dévotion actuellement manifestée par des millions de supporters à l'égard d'individus aussi 'capricieux,' sinon lunatiques, qu'un Donald Trump, un Boris Johnson ou un Jair Bolsonaro (au Brésil) relève du même régime.

Bien entendu, il n'est aucune forme, passée ou actuelle, de totalitarisme ou d'absolutisme qui ne soit aujourd'hui formellement rejetée par l'ensemble des partis ou mouvements politiques: tout le monde, dictateurs inclus et populistes compris, se proclame 'démocrate.' Ce terme, il est vrai, n'est pas en lui-même d'une grande clarté puisqu' on a connu naguère des 'démocraties populaires' à caractère typiquement totalitaire et que se multiplient à présent des 'démocraties illibérales' à forte tendance ab-

${ }^{7}$ Sur la logique sémiotique du piège numérique, cf. Landowski (2016). 
solutiste (comme en Hongrie, en Pologne ou en Italie). Mais dans la conjoncture politique actuelle, le débat se concentre essentiellement autour de la distinction entre deux formes de 'démocratie,' l'une 'représentative,' l'autre dite 'directe,' c'est-à-dire qui, de nos jours, moyens informatiques aidant, permettrait une participation de tous les citoyens à la prise de décision sans passer par l'intermédiaire de représentants élus. Comme on sait, les populistes se déclarent expressément les adversaires de la première forme et se proclament les promoteurs de la seconde.

Nous l'avons déjà noté, la démocratie représentative, telle que traditionnellement pratiquée dans les pays occidentaux, constitue, en termes de sémiotique de l'interaction, un accomplissement parfait du régime de la manipulation. Est-ce à dire que, du seul fait qu'ils s'opposent à cette forme de démocratie et prétendent instaurer à sa place une démocratie directe, les populistes mènent une politique qui relève sémiotiquement du régime interactionnel de l'ajustement ? Pris à la lettre, le modèle ci-dessus pourrait le laisser croire. Pourtant, la réponse ne peut en réalité être que négative. Voilà qui demande quelques explications.

\section{Danger de l'esthésie}

Nous croyons qu'une forme d'organisation de la vie politique qui mette en œuvre une syntaxe interactionnelle correspondant à ce que nous appelons 'l'ajustement' est bel et bien possible. A nos yeux, c'est même cela qui mériterait la noble appellation de 'démocratie directe.' Mais la forme de démocratie proposée par les populistes n'en est que la caricature. Que recouvre donc au juste le concept d'ajustement, et en quoi la démagogie populiste le dénature-t-elle?

L'ajustement, tel que nous l'entendons, ne doit pas être confondu avec l'adaptation (ou l'accomodation), qui relève de la programmation - régime où un actant ne peut agir sur, avec ou contre un autre qu'à condition de respecter les régularités qui régissent son comportement. Il ne consiste pas non plus, comme la manipulation, à faire en sorte que l'un des actants accepte de se plier, fût-ce de mauvais gré, à la volonté de l'autre, car l'ajustement est un régime d'interaction entre égaux; de plus, à la différence du manipulateur, sujet intentionnel qui sait d'avance ce qu'il veut et manipule autrui dans la ferme intention de l'obtenir, le sujet de l'ajustement est fondamentalement un sujet disponible. Par 'disponibilité' nous entendons une disposition actantielle et modale tournée non pas vers l'appropriation du monde mais vers l'accomplissement du potentiel des choses, des gens, et de soi-même. Un sujet 'disponible' ne convoite donc a priori rien de particulier, ni pouvoirs ni honneurs, ni femmes ni richesses... Il aspire seulement à vivre et si possible s'épanouir: c'est un être ouvert à tout ce qui se présente, au présent même et à autrui. 
Lorsqu'entre deux partenaires une telle disponibilité est réciproque, leurs rapports peuvent prendre la forme d'un processus de co-création de sens et pour ainsi dire de réinvention de la vie. N'étant plus exhaustivement encadrée par de rigides déterminismes ou des règles sociales contraignantes (comme dans la programmation), pas non plus entièrement livrée à l'aléa (comme sous le régime de l'accident-assentiment), ni même constamment suspendue à de difficiles négociations entre des intentions divergentes ou des intérêts opposés (comme dans la manipulation), la vie peut alors être conçue, ou pour le moins rêvée, comme une aventure gratifiante - libre et créative une aventure où, dans l'immanence des rapports entre des entités interdépendantes et sensibles les unes aux autres en même temps que respectueuses de leur autonomie respective, chacun cherche à s'accomplir moyennant l'accomplissement corrélatif du potentiel propre à l'autre ou aux autres. Si cela s'applique si bien, par exemple, à l'amitié, c'est parce que l'amitié met en jeu une dynamique d'incitations réciproques grâce auxquelles les partenaires créent entre eux un rapport sans visées ni règles préétablies mais permettant l'invention d'un mode d'être, de penser et de vivre ensemble à caractère inédit (Landowski 2008). Sous sa forme idéale, voilà ce qu'est 'l'ajustement' entre des sujets 'disponibles.'

On se doute qu'un tel idéal est loin d'avoir cours dans l'univers autoritariste et xénophobe du populisme! De nombreuses études sémiotiques ont pourtant montré qu'un tel régime est applicable au fonctionnement de la vie sociale, économique et politique. Une des plus récentes parmi ces études montre (sans utiliser le terme même d'ajustement) quelle révolution culturelle et politique est susceptible d'introduire dans le fonctionnement social des organes de production économique et dans le vécu quotidien de leurs agents, le recours à une telle syntaxe (Fontanille 2018). D'autres travaux indiquent à quelles conditions il peut en aller de même dans des domaines de la pratique les plus divers, du sport au design (Demuru 2015; Scóz 2018), de la recherche à la prière (Petitimbert 2017, 2015), ou encore en matière de gestion de collectivités publiques ou privées (Ciaco 2013; Cervelli 2013; Petitimbert 2013; Catellani 2018). Débouchant sur une écologie du sens, la logique du régime d'ajustement offre même, selon nous, une voie de réflexion alternative sur les dramatiques problèmes environnementaux et sociétaux de notre temps (Landowski 2017; Calame 2015).

Mais que ce soit sur le plan microsocial ou sur un plan plus large, les formes de démocratie directe envisagées par ces diverses études ne sont possibles qu'à condition d'observer à la fois les deux principes dont une syntaxe d'ajustement suppose la conjonction, celui de sensibilité, certes, mais aussi celui de disponibilité. Or, du régime d'ajustement ainsi défini, les populistes ne prennent que ce qui leur convient, à savoir le seul côté sensible. Et s'en remettre exclusivement à la contagion du sentir et à l'emprise psychologique que procure une présence de chaque instant sur les réseaux sociaux, c'est ouvrir la voie aux pires formes d'asservissement en transformant la 
collectivité en une 'masse thymique' indifférenciée, amorphe et malléable. Comme on dit, le tribun 'électrise' les foules: son 'fil conducteur,' ce sont les corps à travers lesquels passe sa puissance énergétique, si bien que d'un corps à l'autre la ferveur populaire se propage d'elle-même dans tous les azimuts. En fait de 'démocratie directe,' il ne reste plus alors qu'un slogan à valeur connotative, trompeur qui plus est.

Aussi, quand nous avons introduit, sous le nom de 'contagion,' la dimension sensible parmi les variables de la relation intersubjective, nous avons d'emblée distingué à son propos deux usages possibles, bilatéral ou unilatéral (Landowski 2004: 130-133). L'usage unilatéral, c'est l'annihilation de l'autre en tant que sujet, par absorption ou fusion. L'exploitation du sensible peut alors être mise au service de la 'manipulation,' non pas seulement au sens établi par la sémiotique narrative (où elle engage la persuasion et débouche sur le contrat) mais aussi selon l'acception usuelle et péjorative: soumettre l'autre à soi en jouant sur son corps, comme dans l'hypnose qui réduit l'autre à un non-sujet programmé, ou sur ses pulsions, telle la publicité qui 'fait désirer' par contagion sensible (Landowski 2007).

La propagande actuelle ne fait pas autre chose. Misant tout sur l'esthésie au point d'arriver à faire passer les fake news les plus rocambolesques pour des vérités établies, elle paraît neutraliser les facultés de raisonnement de beaucoup de nos contemporains. Il n'en reste pas moins que contrairement à ce que la candidate démocrate à l'élection présidentielle nord-américaine de 2016, Hillary Clinton, eut un jour la maladresse d'insinuer, les électeurs séduits par la rhétorique de bateleur et les clins d'œil de son adversaire n'étaient pas, constitutivement, moins capables de jugement que les autres : eux aussi, ils étaient, comme nous tous, à la fois sensibles et intelligents. Le fait qu'ils aient été esthésiquement réceptifs face aux charmes incantatoires (et capillaires) de leur héros alors que, corrélativement, ils ne pouvaient pas supporter l'hexis 'bon genre' de sa rivale, sa morgue de femme en tous points 'comme il faut' (de la coiffure à la chaussure), ne les empêchait pas, par ailleurs, d'évaluer cognitivement divers aspects moins 'épidermiques' de la situation. Et pourtant, en l'occurrence, c'est une logique du sensible suspendant la décision au jeu des attractions et des répulsions immédiates qui a prévalu sur le poids des arguments.

Le fait que le cognitif et l'esthésique, loin de s'exclure l'un l'autre, soient au contraire, en théorie, conjointement présents à la base de tous nos choix, y compris politiques, n'empêche donc pas que néanmoins, dans certains contextes, ils tendent à se neutraliser l'un l'autre. C'est ce qui permet de comprendre qu'une campagne électorale et même une stratégie de communication à long terme puissent, au choix, s'appuyer préférentiellement sur l'une ou l'autre de ces deux facettes de notre compréhension du monde. Mais on ne parlerait pas de populisme si les grands et les petits bouffons de notre temps n'avaient pas depuis longtemps fait à cet égard leur choix. 


\section{Ouvrages cités}

Calame, Claude 2015. Avenir de la planète et urgence climatique. Au-delà de l'opposition nature / culture. Fécamp: Lignes.

Catellani, Andrea 2018. L'entreprise responsable et ses parties prenantes: entre 'manipulation' et co-construction de sens. Actes Sémiotiques 122. (https: / / bit.ly/2OOcm1c).

Cervelli, Pierluigi 2013. Fallimenti della programmazione e dinamiche dell'aggiustamento. Sull'auto-produzione dello spazio pubblico in una periferia di Roma. In: A.C. de Oliveira (éd.) As Interações sensiveis. Ensaios de sociossemiótica. São Paulo: Estação das Letras e Cores.

Ciaco, João 2013. A inovação em discursos publicitários: semiótica e marketing. São Paulo: Estação das Letras e Cores.

Demuru, Paolo 2015. Malandragem vs Arte di arrangiarsi: Stili di vita e forme dell'aggiustamento tra Brasile e Italia. Actes Sémiotiques 118 (https: / bit.ly/3sfGlhg).

Fontanille, Jacques 2018. La coopérative et son territoire. In: Nicolas Couegnas \& Jacques Fontanille (dir.) Terres de sens. Essai d'anthroposémiotique. Limoges: Pulim.

Greimas, Agirdas J. 1987. De l'Imperfection. Périgueux: Fanlac.

Landowski, Eric 1997. Présences de l'autre. Paris: P.U.F.

Landowski, Eric 2004. Passions sans nom. Paris: P.U.F.

Landowski, Eric 2007. Le triangle émotionnel de la publicité. Semiotica 163 (1/4): 59-73.

Landowski, Eric 2008. L'épreuve de l'autre. Sign Systems Studies 34(2): 317-336.

Landowski, Eric 2016. Pièges: de la prise de corps à la mise en ligne. Carte Semiotiche-Annali 4: 20-40.

Landowski, Eric 2017. Petit manifeste sémiotique en l'honneur et à l'attention du camarade sociologue Pekka Sulkunen. Actes Sémiotiques 120 (https: / / bit.ly /3qGn73J).

Landowski, Eric 2019. Politiques de la sémiotique. Rivista Italiana di Filosofia del Linguaggio 13 (2): 6-25 (https: / / bit.ly /3pKIlfO).

Petitimbert, Jean-Paul 2013. Entre l'ordre et le chaos: la précarité comme stratégie d'entreprise. Actes Sémiotiques 116 (https: / / bit.ly/3ulkcQu).

Petitimbert, Jean-Paul 2015. Lecture sémiotique d'une pratique et d'une interaction particulière: 1'hésychasme orthodoxe. Actes Sémiotiques 118 (https: / / bit.ly/3aDpZsJ).

Petitimbert, Jean-Paul 2017. Régimes de sens et logique des sciences. Interactions sociosémiotiques et avancées scientifiques. Actes Sémiotiques 120 (https: / / bit.ly / 3aDCkgz).

Scóz, Murilo 2018. Por uma abordagem sociossemiótica do design de interação. Actes Sémiotiques 121 (https:/ / bit.ly/37wXPh9).

\section{AUTEUR}

Eric Landowski Research director CNRS, Paris. Visiting Professor at the University of São Paulo, Brazil (PUC-SP) and the University of Vilnius, Lithuania. Director of the Centre for Sociosemiotic Research (PUC-SP). 


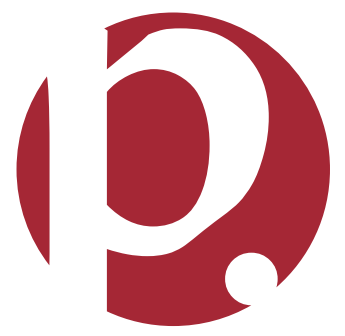

